of neuroleptic treatment. Examination of the subjects shows that actually, those with TD have twofold more psychiatric admissions compared with those without TD; thus they are probably the more severely ill group of patients. It is to be expected that they might have received higher neuroleptic dosage during hospital stays, and so the cumulative neuroleptic dosage might have been higher in the group with TD. The lower current neuroleptic dosage in the group with TD compared with those without TD may reflect the attending doctors' attempts to reduce dosage of neuroleptics after onset of TD, rather than these patients requiring lower maintenance dose of neuroleptics. To establish that lithium exposure is really a risk factor for the development of TD, the cumulative neuroleptic dosage should be matched for the two groups of patients.

Department of Psychiatry

Helen Chiu

Chinese University

Prince of Wales Hospital

Shatin, Hong Kong

SIR: Dr Falvey is clearly correct in pointing out the multivariate aetiology of TD. The disease process, ageing and psychotropic medication probably all interact to produce the disorder. Our study, in attempting to elucidate the role of lithium in the development of TD in bipolar affective disorder, is in no way suggesting a univariate iatrogenic model for TD. The literature to date does not support such a model, and such thinking has considerable medico-legal significance.

It is possible, as Dr Chiu argues, that the lower current neuroleptic dosage in the group with TD may be an attempt by the clinician to reduce the dosage of neuroleptics after the onset of TD. A review of the patient records in general does not support such a view. In the majority of patients, the presence of even moderately severe orofacial dyskinesia was not noted by the attending clinician.

The fact that all patients fulfilled Schooler \& Kane research diagnostic criteria for TD meant that each had at least 2 months' neuroleptic exposure. We agree with Dr Volavka that a study of patients treated with lithium but having no previous neuroleptic exposure would be useful. Such patients are, however, few and far between.

Timothy G. DinaN DORA KOHEN

\author{
St James's Hospital \\ James's Street \\ P.O. Box 580 \\ Dublin 8
}

\section{The opiate prescribing debate}

SIR: Your readers will have been mislead by a recent letter from Dr John Marks (Journal, October 1989. $155,566)$ in which he suggests that making opiate drugs more available reduces the problems associated with drugs. Dr Marks cites two references and suggests that these support his assertion.

Unfortunately these references are not to clinical trials or studies, but correspondence in which he restates his well-known views. The relationship between prescribing policies and the behaviour of drug users is far from understood. The notion that more liberal prescribing policies will reduce the crime rate remains an important hypothesis which requires testing.

\author{
St George's Hospital Medical School \\ Department of Psychiatry \\ Cranmer Terrace \\ London SW17 ORE
}

ANDREW JOHNS

\section{The evaluation of mental health care systems}

SIR: Häfner \& an der Heiden (Journal, July 1989, 155, 12-17) discuss the evaluation of mental health care systems. They leave a number of questions unanswered and, indeed, unasked.

The question is set as to whether out-patient care affected either the length of time spent in the community or the length of time during readmission to hospital. There is inadequate description of the nature of this care, and no real mention of the alternatives, this despite the earlier plea that "interventions subject to evaluation must be described precisely".

It is debatable whether the two effectiveness criteria cited constitute a valid therapeutic outcome when used in such an unqualified way: perhaps the reasons for readmission would shed some light on how out-patient care is provided so cheaply in this case? Similarly, Fig. 2 of the paper raises the question as to why patients with the highest chance of readmission had the lowest frequency of out-patient contact: surely it is wrong to conclude that frequency of out-patient contact directly influences the chance of readmission, despite allowing for a few of the possible intervening variables (symptoms, length of previous in-patient stay, and living conditions)?

Our main comments are reserved for the method of economic analysis (itself at odds with the request not to overvalue economic factors). Direct monetary cost is used as the sole indicator of total cost and, subsequently, an attempt is made to relate this to (unmeasured) non-monetary costs such as burden of 
care: this relationship cannot be assumed to be so clear-cut. There is minimal description of how the average cost per patient was calculated, yet this is given inordinate prominence in concluding (wholly on grounds of cost) which patients are best treated in hospitals - which, by the authors' own admission, are in a state of "scandalous neglect" and presumably in need of significant capital investment. Indeed, no mention is made of whether costings differentiated between capital and revenue expenditure, an essential distinction in any cost analysis.

The system of mental health care analysed appears to be undergoing evolutionary rather than radical change, yet average cost per patient is used rather than marginal cost (cost per extra patient within an existing system). This is a particularly misleading omission, because the cost of maintaining a small number of highly dependent patients (8 people, or $6 \%$ of the cohort) in the community is compared with the average cost of in-patient care, despite the likelihood that such patients will incur aboveaverage costs in hospital. In any case, Fig. 4 of the paper indicates that such patients could be incorporated within a community care system with a relatively small increase in average cost.

This paper has stepped into a methodological minefield, and we conclude that it has failed to support the justifiable caution about rushing headlong into community care. Nevertheless, it emphatically exposes the need for good economic evaluation in psychiatry.

\section{St George's Hospital \\ Department of Adult Psychiatry \\ Clare House \\ Blackshaw Road \\ London SW170QT}

SIR: Drs Checinski \& Goddard criticise the approach to cost analysis in our paper. If the analysis of costeffectiveness of a mental health care system had been the central issue of study, this criticism would be justified. The purpose of our study, however, was to evaluate the effectiveness of a specific component of extramural care in a representative cohort of schizophrenic patients, on the basis of an observational study with a naturalistic design. We substituted for the lack of control by a methodological approach that by means of a specific model (Fig. 1 in our paper) permitted the partialing out of confounding influences - taking into account the time sequence on the interesting independent and dependent variables. The description of average direct costs and costs per case was meant to demonstrate that in principle, the actual structure and politics of care form the background of calculating these values, which for this reason cannot simply be applied to a different system. In the system evaluated, providing mental health care for the Mannheim population, the situation is characterised by the fact that about $25 \%$ of the schizophrenic patients needing longterm ( $>1$ year) residential care were still in continued hospital care. The monotonically increasing costs per case (Fig. 4 of our paper) illustrate this, inasmuch as the curve of increasing costs per case would presumably continue to ascend if these $25 \%$ of patients were reduced, and consequently the number of cases exceeding the threshold value of the comparable costs for in-patient treatment increased. However, for describing this issue the calculation of the marginal costs would be of minor significance.

The majority of studies do not consider the interdependence between the costs of extramural care and the proportion of patients in hospital and complementary care. Doubtlessly we have ventured into a methodological minefield, but only to attract attention to some unnoticed mines. We leave it to Drs Checinski \& Goddard to sweep them.

Contrary to Drs Checinski \& Goddard's postulation, we found that if we considered capital investment in a very small number of heterogeneous facilities serving our catchment area, which has a population of 300000 , this would have resulted in considerable distortion. The determination of the total direct costs of medical care, social care, etc. proved to be the only comparable index for case-related costs of care.

Indeed, the analogy drawn between monetary and non-monetary costs is not based on empirical data - the collection of which was not the subject of our study. By indicating the tendency of a parallel course for these two types of costs, we intended to point out that due to the reduction of numbers of psychiatric beds, severely disordered patients would also have to be discharged. Extramural care for them would not be cheaper, and presumably would be worse than long-term admission. There is no doubt that in principle, more severely disordered patients also cause higher costs when cared for in hospital. However, these increased costs did not arise in a measurable way, since they had no effect on the number of staff or on the equipment of the hospitals concerned. This means that additional care of the more severely disordered patients was provided at the expense of the less severely disordered. The problems involved could not be treated in our paper. 